Commun. math. Phys. 37, 335-341 (1974)

(C) by Springer-Verlag 1974

\title{
A Family of Jordan-Brans-Dicke Kerr Solutions
}

\author{
C. B. G. McIntosh \\ Mathematics Department, Monash University, Clayton, Victoria, Australia
}

Received November 23, 1973; in revised form May 6, 1974

\begin{abstract}
A family of solutions of the vacuum Jordan-Brans-Dicke or scalar-tensor gravitational field equations is given. This family reduces to the Kerr rotating solution of the vacuum Einstein equations when the scalar field is constant. The family does not have spherical symmetry when the rotation is zero and the scalar field is not constant. The method used to generate the new solutions can also be used to obtain vacuum Jordan-Brans-Dicke solutions from any given vacuum stationary, axisymmetric solution.
\end{abstract}

\section{Introduction}

Heckmann, Jordan and Fricke [1] and Brans [2] have discussed static spherically symmetric solutions of the vacuum Jordan-Brans-Dicke [JBD] gravitational field equations. The first solution in the list of four families of solutions which Brans gives is a generalisation of the Schwarzschild solution of the vacuum Einstein field equations but has the property that the event horizon of the Schwarzschild solution becomes a singular surface and may not be termed a "black hole". The Brans solution I was later discussed in a different context and with different interpretation by Janis et al. [3] (see also [4-6]). Charged versions of Brans' solutions were recently given by Buchdahl [7] and Luke and Szamosi [8].

Hawking [9] and Johnson [10] (cf. Thorne and Dykla [11]) say that the only black holes in the JBD theory [12] are Einstein black holes. This means that the Kerr family of solutions with constant scalar field are the only stationary black hole solutions of the vacuum JBD equations provided that the Carter conjecture is true. Nariai [5] suggests that the lack of black holes solutions with non-constant scalar field means that the JBD theory gives quite different predictions for gravitational collapse to those in the Einstein theory.

So far there have been no exact rotating solutions of the vacuum JBD equations which reduce to the Kerr solution when the scalar field is zero. A family of such solutions is given in this paper. They are axisymmetric and stationary but do not reduce in the static case (when 
the rotation is zero) to Brans' solution I as may be expected but to nonspherically symmetric solutions in a family discussed by Penney [13].

The method of generation of the new vacuum JBD solutions from the Kerr solution discussed here works also with any vacuum stationary axisymmetric solution other than the Kerr solution taken as the basic solution.

\section{Basic Equations}

The vacuum JBD field equations can be written as

$$
R_{\mu \nu}=-f \Phi,{ }_{\mu} \Phi,{ }_{v} .
$$

Here $\Phi$ is a scalar field, $f$ a coupling constant, proportional to $2 \omega+3$ where $\omega$ is the coupling constant of the JBD theory, and the conformal frame being used is the "Einstein frame" as discussed by Dicke [14] (see also [9]). The field Eq. (1) also arise when a massless scalar field is coupled to the gravitational field in Einstein's theory and when there are no other source terms present. The Bianchi identities give for Eq. (1) the wave equation

$$
\square \Phi=0
$$

so that the extra field equation in the JBD theory is automatically satisfied in the vacuum case.

Solutions of (1) will only be sought for a stationary, axisymmetric space, in which case the metric can always be written as

$$
d s^{2}=e^{2 v} d t^{2}-e^{2 \psi}(d \phi-\omega d t)^{2}-e^{2 \alpha}\left(d x^{2}\right)^{2}-e^{2 \beta}\left(d x^{3}\right)^{2}
$$

where $v, \psi, \omega, \alpha$, and $\beta$ are functions of $x^{2}$ and $x^{3}$ only, and where the scalar field is a function of $x^{2}$ and $x^{3}$ only, that is

$$
\Phi=\Phi\left(x^{2}, x^{3}\right) .
$$

There is coordinate freedom in the metric to restrict $\alpha$ and $\beta$ by a coordinate condition such as the condition

$$
\alpha=\beta
$$

which will be applied through most of this paper.

With

$$
x^{\mu}=(t, \phi, R, \theta),
$$


the field equations can be written down from Chandrasekhar and Friedman [15] as

$$
\begin{aligned}
-f \Phi_{R}^{2}= & \beta_{R R}+\beta_{\theta \theta}-\beta_{R}(v+\psi)_{R}+\beta_{\theta}(v+\psi)_{\theta}+(v+\psi)_{R R} \\
& +v_{R}^{2}+\psi_{R}^{2}-\frac{1}{2} e^{2 \psi-2 v} \omega_{R}^{2} \\
-f \Phi_{R} \Phi_{\theta}= & (v+\psi)_{R \theta}+v_{R} v_{\theta}+\psi_{R} \psi_{\theta}-\beta_{R}(v+\psi)_{\theta}-\beta_{\theta}(v+\psi)_{R} \\
& -\frac{1}{2} e^{2 \psi-2 v} \omega_{R} \omega_{\theta} \\
-f \Phi_{\theta}^{2}= & \beta_{R R}+\beta_{\theta \theta}+\beta_{R}(v+\psi)_{R}-\beta_{\theta}(v+\psi)_{\theta}+(v+\psi)_{\theta \theta} \\
& +v_{\theta}^{2}+\psi_{\theta}^{2}-\frac{1}{2} e^{2 \psi-2 v} \omega_{\theta}^{2} \\
0= & \psi_{R R}+\psi_{\theta \theta}+\psi_{R}(v+\psi)_{R}+\psi_{\theta}(v+\psi)_{\theta} \\
& +\frac{1}{2} e^{2 \psi-2 v}\left(\omega_{R}^{2}+\omega_{\theta}^{2}\right) \\
0= & \omega_{R R}+\omega_{R}(3 \psi-v)_{R}+\omega_{\theta \theta}+\omega_{\theta}(3 \psi-v)_{\theta} \\
0= & \left(e^{v+\psi}\right)_{R R}+\left(e^{v+\psi}\right)_{\theta \theta} .
\end{aligned}
$$

The last three equations are, respectively, the $R_{11}$, the $R_{1}^{0}$ and the $R_{0}^{0}+R_{1}^{1}$ equations. Any non-constant solution of (7f) may be chosen and is equivalent to any other non-constant solution through a coordinate change (see [16]). The solution taken here is

where

$$
\exp (v+\psi)=\Gamma(R) \sin \theta
$$

$$
\Gamma(R)=e^{R}-\frac{1}{4}\left(M^{2}-a^{2}\right) e^{-R}
$$

and where $M$ and $a$ are constants.

\section{Brans' Solution I}

For Brans' solution I to be written as a solution of the field Eq. (7), a conformal mapping and a coordinate change must be made to the form as given by Brans [2]. The solution then becomes

$$
\begin{aligned}
\beta & =\beta_{0}-R+(1+D) \ln \left|e^{R}+\frac{1}{2} M\right|+(1-D) \ln \left|e^{R}-\frac{1}{2} M\right| \\
v & =v_{0}+D \ln \left|e^{R}-\frac{1}{2} M\right|-D \ln \left|e^{R}+\frac{1}{2} M\right| \\
\psi & =\beta+\ln |\sin \theta|-\left(\beta_{0}+v_{0}\right) \\
\Phi & =\Phi_{0} \pm\left\{\left(1-D^{2}\right) / 2 f\right\}^{\frac{1}{2}} \ln \left|\left(e^{R}-\frac{1}{2} M\right) /\left(e^{R}+\frac{1}{2} M\right)\right|
\end{aligned}
$$

where $\beta_{0}, v_{0}, \Phi_{0}$ and $D$ are constants. When $D=1$ the solution is the Schwarzschild solution. It follows that the solution may be written as

$$
\begin{gathered}
\beta(R)=\beta_{S}(R)+\tilde{\beta}(R) \\
v(R)=v_{S}(R)-\tilde{\beta}(R) \\
\psi(R, \theta)=\psi_{S}(R, \theta)+\tilde{\beta}(R)
\end{gathered}
$$


where the subscript $S$ denotes the equivalent term in the Schwarzschild solution and $\tilde{\beta}$ is an extra variable which must be added to the "S" variables to obtain the new solution. The $e^{2 v} d t^{2}$ part of the metric is thus multiplied by $e^{-2 \tilde{\beta}}$ and the remaining three-space part by its inverse. $\psi+v$ remains unchanged. When looked at with Eq. (7) as the starting point, the addition works because the vacuum Eq. $\left(7 \mathrm{~b}-\mathrm{f}\right.$ ) (with $\Phi_{\theta}=0$, $\omega=0)$ are linear in the $\tilde{\beta}$ variable and give

$$
0=\tilde{\beta}_{R R}+\tilde{\beta}_{R} \Gamma^{\prime} / \Gamma+\text { Schwarzschild terms }
$$

where the contribution of the Schwarzschild terms is obviously zero. After these equations are solved for $\tilde{\beta},(7 \mathrm{a})$ is solved for $\Phi$.

\section{Rotating Solutions}

The approach as outlined in the last part of Section 3 does not carry over directly into the case of a rotating metric because of the presence of the $\omega$ terms in Eq. (7). However if only the change

$$
\beta(R, \theta)=\beta_{K}(R, \theta)+\tilde{\beta}(R, \theta)
$$

is made where $\beta_{K}$ is the value of $\beta$ for some known vacuum solution, $\tilde{\beta}$ is some extra variable, and $v, \psi$, and $\omega$ keep their forms for this known metric, then the field Eq. (7) give

$$
\begin{aligned}
-f \Phi_{R}^{2} & =\tilde{\beta}_{R R}+\tilde{\beta}_{\theta \theta}-\tilde{\beta}_{R} \Gamma^{\prime} / \Gamma+\tilde{\beta}_{\theta} \cot \theta \\
-f \Phi_{R} \Phi_{\theta} & =-\tilde{\beta}_{R} \cot \theta-\tilde{\beta}_{\theta} \Gamma^{\prime} / \Gamma \\
-f \Phi_{\theta}^{2} & =\tilde{\beta}_{R R}+\tilde{\beta}_{\theta \theta}+\tilde{\beta}_{R} \Gamma^{\prime} / \Gamma-\tilde{\beta}_{\theta} \cot \theta .
\end{aligned}
$$

Thus the geometry within the group orbits (generated by $\partial / \partial t$ and $\partial / \partial \phi)$ is left unchanged; only the complementary $(R, \theta)$ surfaces are deformed. When Eq. (14) are solved for some $\tilde{\beta}$ and $\Phi$ and $\beta$ formed from (13), then a rotating solution which satisfied (1) has been found. $\Phi$ will satisfy (2) or

$$
\Phi_{R R}+\Phi_{\theta \theta}+\Phi_{R} \Gamma^{\prime} / \Gamma+\Phi_{\theta} \cot \theta=0 .
$$

Conversely, where a solution $\Phi$ of (15) is known, $\tilde{\beta}$ can be found from (14), that is from

$$
\begin{aligned}
& \tilde{\beta}_{R}\left(\Gamma^{\prime 2} / \Gamma^{2}+\cot ^{2} \theta\right)=f\left[\Phi_{R} \Phi_{\theta} \cot \theta+\frac{1}{2}\left(\Phi_{R}^{2}-\Phi_{\theta}^{2}\right) \Gamma^{\prime} / \Gamma\right] \\
& \tilde{\beta}_{\theta}\left(\Gamma^{\prime 2} / \Gamma^{2}+\cot ^{2} \theta\right)=f\left[\Phi_{R} \Phi_{\theta} \Gamma^{\prime} / \Gamma-\frac{1}{2}\left(\Phi_{R}^{2}-\Phi_{\theta}^{2}\right) \cot \theta\right] .
\end{aligned}
$$

The integrability condition for $\tilde{\beta}$ is satisfied by (16); thus for every solution $\Phi$ of (15), a solution $\tilde{\beta}$ of (16) exists and thus a new vacuum $\mathrm{JBD}$ or scalar-tensor solution may be found. 
The most relevant solution is probably that in the case when $\Phi$ depends on $R$ only. Then, from (15),

$$
\Phi=\Phi_{0}+A \ln \left|\frac{e^{R}-\frac{1}{2}\left(M^{2}-a^{2}\right)^{\frac{1}{2}}}{e^{R}+\frac{1}{2}\left(M^{2}-a^{2}\right)^{\frac{1}{2}}}\right|
$$

where $A$ and $\Phi_{0}$ are constants. Notice that $\Phi$ tends to $\Phi_{0}$ as $R$ tends to infinity and that $\Phi$ is singular on $\Gamma=0$. Equation (16) now gives

$$
\tilde{\beta}=\tilde{\beta}_{0}+f A^{2} \ln \left|\Gamma^{2} /\left\{\Gamma^{2}+\left(M^{2}-a^{2}\right) \sin ^{2} \theta\right\}\right|
$$

where $\tilde{\beta}_{0}$ is a constant.

There is a corresponding solution when $\Phi$ depends on $\theta$ only.

Another solution of (15) and (16) is

$$
\tilde{\beta}=\tilde{\beta}_{0}+A(\Gamma \sin \theta)^{2}, \quad \Phi=\Phi_{0} \pm 2(-A / f)^{\frac{1}{2}} \Gamma^{\prime} \cos \theta
$$

which is interesting in that with $A$ negative and hence $f$ positive, the metric will in general be asymptotically flat if the "background" vacuum metric is and the curvature invariant will not become infinite at any other values of $R$ and $\theta$ other than where the corresponding invariant of the background metric does. The scalar field however becomes infinite for infinite $R$.

If $\Phi$ is constant, the only solution for $\tilde{\beta}$ is $\tilde{\beta}=$ constant; that is no new vacuum solutions can be formed with constant $\Phi$.

\section{Jordan-Brans-Dicke-Kerr Solutions}

The Kerr solution, because it is a stationary and axisymmetric vacuum solution, can have a scalar field "added" onto it in the way discussed in Section 4. In the coordinates (3), the Kerr metric has been given by Boyer and Lindquist [17] as

where

$$
\begin{gathered}
e^{2 \psi}=\sin ^{2} \theta P / \Sigma, \quad e^{2 v}=\Sigma \Delta / P, \quad \omega=2 \mathrm{Mar} / P \\
e^{2 \alpha}=\Sigma / \Delta, \quad e^{2 \beta}=\Sigma
\end{gathered}
$$

$$
\begin{gathered}
\Sigma=r^{2}+a^{2} \cos ^{2} \theta, \quad \Delta=r^{2}+a^{2}-2 M r, \\
P=\Delta \Sigma+2 M r\left(r^{2}+a^{2}\right) .
\end{gathered}
$$

When the coordinates are changed so that $\alpha=\beta$,

$$
\begin{gathered}
e^{2 \alpha}=e^{2 \beta}=\Sigma(R), \\
\Delta(r)=\Gamma^{2}(R)=\left\{e^{R}-\frac{1}{4}\left(M^{2}-a^{2}\right) e^{-R}\right\}^{2}
\end{gathered}
$$

and $\psi, v$ and, $\omega$ can be calculated immediately as functions of $R . \Delta=\Gamma^{2}$ can be solved for $r$ as

$$
r=e^{R}+M+\frac{1}{4}\left(M^{2}-a^{2}\right) e^{-R} .
$$


New solutions which are either "vacuum Kerr-Jordan-Brans-Dicke" solutions or "scalar-tensor-Kerr" solutions can then be obtained by multiplying the $e^{2 \beta}\left(d R^{2}+d \theta^{2}\right)$ section of the Kerr metric by $e^{2 \widetilde{\beta}}$ where $\tilde{\beta}$ is given by solving (15) and (16) and by keeping the rest of the metric unchanged.

Consider the solution with $\Phi$ a function of $R$ only, $|M|>|a|$, and $\Phi$ and $\tilde{\beta}$ given by (17) and (18), but with $\tilde{\beta}_{0}=0$. Then

$$
\Phi=\Phi_{0}+\frac{1}{2} A \ln \left|\left(r-r_{+}\right) /\left(r-r_{-}\right)\right|
$$

where $r_{+}$and $r_{-}$are the two solutions of $\Delta=0$, namely

and

$$
r_{ \pm}=M \pm\left(M^{2}-a^{2}\right)^{\frac{1}{2}}
$$

$$
\tilde{\beta}=f A^{2} \ln \left|\frac{(r-M)^{2}-\left(M^{2}-a^{2}\right)}{(r-M)^{2}-\left(M^{2}-a^{2}\right) \cos ^{2} \theta}\right| .
$$

Equation (24) can be written for large $r$ as

$$
\Phi=\Phi_{0}-A\left(M^{2}-a^{2}\right)^{\frac{1}{2}} / r+0\left(r^{-2}\right) .
$$

This shows the sense in which $A$ is the "scalar charge" of the solution. $M$ and $a$ are the mass and specific angular momentum respectively. In these coordinates, both $e^{2 \alpha}$ and $e^{2 \beta}$ are multiplied by $e^{2 \tilde{\beta}}$ for the new solution to be obtained.

The curvature invariant $R$ becomes infinite on $\Gamma=0$ for $f>0$ (equivalent to $\omega>-\frac{3}{2}$ ) which is the expected value of $f$ in the JBD theory. The Kerr metric event horizons $r=r_{+}$and $r=r_{-}$then both become singular surfaces. $\Phi$ is singular on each of these surfaces. As $r$ tends to infinity, $\Phi$ is asymptotically constant and the metric is asymptotically flat. This solution with $a=0$ (no rotation) is not the Brans - I solution but is a member of a family of solutions discussed by Penney [13] [his Eq. (12) with $A=1$ ] and is not spherically symmetric.

\section{Discussion}

Any stationary and axisymmetric vacuum solution, including the Kerr solution, can be used to generate a new family of vacuum JBD or scalar-tensor solutions by using the method discussed in Section 4. Other work on generating new solutions in the vacuum and JBD cases from given vacuum solutions has been discussed by Geroch $[18,19]$, Buchdahl [20] and other writers. The relationships between these methods has been discussed by Sneddon and the author [21]. 
Acknowledgements. I wish to thank Professor R. P. Kerr and his colleagues of the mathematics department, University of Canterbury, Christchurch, New Zealand, for their very kind hospitality while much of this work was undertaken. I would also like to thank Professor Kerr, Dr. W. B. Wilson, Dr. E. D. Fackerell, A. W.-C. Lun and G. E. Sneddon for useful discussions concerning this work. Finally I thank Professor Ehlers for making a number of useful criticisms on an earlier draft of this paper.

\section{References}

1. Heckmann, O., Jordan, P., Fricke, W.: Astrophys. Zeitschr. 28, 113-149 (1951)

2. Brans, C.H.: Phys. Rev. 125, $2194-2201$ (1962)

3. Janis, A.I., Newman, E.T., Winicour,J.: Phys. Rev. Letters 20, 878-880 (1968)

4. Winicour, J., Janis, A.I., Newman, E.T.: Phys. Rev. 176, 1507-1513 (1968)

5. Nariai, H.: Progr. Theoret. Phys. (Kyoto) 42, $742-744$ (1969)

6. Matsuda, T.: Progr. Theoret. Phys. (Kyoto) 47, 738-740 (1972)

7. Buchdahl, H. A.: Nuovo Cim. 12B, 269-287 (1972)

8. Luke,S. K., Szamosi, G.: Phys. Rev. D. 6, 3359-3363 (1972)

9. Hawking, S. W.: Commun. math. Phys. 25, 167-171 (1972)

10. Johnson, M.: Lett. Nuovo Cimento 4, 323-327 (1972)

11. Thorne, K.S., Dykla, J.J.: Ap. J. 166, L35-L38 (1971)

12. Brans, C.H., Dicke, R. H.: Phys. Rev. 124, 925-935 (1961)

13. Penney, R.: Phys. Rev. 174, 1578-1579 (1968)

14. Dicke, R.H.: Phys. Rev. 125, $2163-2167$ (1962)

15. Chandrasekhar, S., Friedman, J.L.: Ap. J. 175, 379-405 (1972)

16. Synge, J.L.: Relativity: The general theory, pp. 309-312. Amsterdam: North-Holland Publishing Co., 1966

17. Boyer, R. H., Linquist, R. W.: J. Math. Phys. 8, 265-281 (1967)

18. Geroch, R.: J. Math. Phys. 12, $918-924$ (1971)

19. Geroch, R.: J. Math. Phys. 13, 394-404 (1972)

20. Buchdahl, H. A.: Int. J. Theor, Phys. 6, 407-412 (1972)

21. Sneddon, G.E., McIntosh, C. B. G.: Aust. J. Phys. 27, 411-416 (1974)

Communicated by J. Ehlers

C. B. G. McIntosh

Department of Mathematics

Monash University

Clayton, Victoria 3168, Australia 
